\title{
Nonlinear Spectrum of Conventional OFDM and WDM Return-to-Zero Signals in Nonlinear Channel
}

\author{
Sergey Turitsyn, Fellow, OSA, Egor Sedov, Alexey Redyuk and Mikhail Fedoruk
}

(Invited Paper)

\begin{abstract}
The nonlinear Schrödinger equation (NLSE) is often used as a master path-average model for fiber-optic links to analyse fundamental properties of such nonlinear communication channels. Transmission of signal in nonlinear channels is conceptually different from linear communications. We use here the NLSE channel model to explain and illustrate some new unusual features introduced by nonlinearity. In general, NLSE describes the co-existence of dispersive (continuous) waves and localised (here in time) waves - soliton pulses. The nonlinear Fourier transform method allows one to compute for any given temporal signal the so-called nonlinear spectrum, that defines both continuous spectrum (analogue to conventional Fourier spectral presentation) and solitonic components. Nonlinear spectrum remains invariant during signal evolution in the NLSE channel. We examine conventional orthogonal frequency-division multiplexing (OFDM) and wavelength-division multiplexing (WDM) return-tozero signals and demonstrate that both signals at certain power levels have soliton component. We would like to stress that this effect is completely different from the soliton communications studied in the past. Applying Zakharov-Shabat spectral problem [1] to a single WDM or OFDM symbol with multiple sub-carriers we quantify the effect of statistical occurrence of discrete eigenvalues in such an information-bearing optical signal. Moreover, we observe that at signal powers optimal for transmission an OFDM symbol with high probability has a soliton component.
\end{abstract}

Index Terms-Nonlinear optics; Communication system nonlinearities; Optical fiber communication; Optical solitons; Wavelength division multiplexing; Subcarrier multiplexing; Simulation; Transmission line theory; Signal analysis.

\section{INTRODUCTION}

$\mathbf{N}$ ONLINEAR effects in optical fiber limit performance of modern optical communication systems at high signal powers. This defines a crucial practical difference between nonlinear and linear communication channels, such as e.g. classical linear additive white Gaussian noise (AWGN) channel, where capacity can be improved by increasing signal power and, respectively, signal-to-noise ratio (SNR). Note, that most of the conventional communication methods and techniques have been designed and developed for linear communication channels. Therefore, it comes as no surprise that nonlinearity imposes undesirable signal distortions when these (linear) communication methods are used in nonlinear fiber channels. In recent years, considerable efforts have been made to reduce the negative impact of nonlinear fiber effects using

S. Turitsyn is with Aston Institute of Photonic Technologies, Aston University, Birmingham B4 7ET, UK, e-mail: s.k.turitsyn@aston.ac.uk.

A. Redyuk and M. Fedoruk are with Institute of Computational Technologies, Siberian Branch, Russian Academy of Sciences, Novosibirsk, 630090, Russia.

S. Turitsyn, A. Redyuk, M. Fedoruk and E. Sedov are with Novosibirsk State University, Novosibirsk, 630090, Russia. various compensation methods (see e.g. for more detail [2][9] and references therein). However, channel nonlinearity potentially can also offer new interesting and unconventional techniques, such as e.g. nonlinear Fourier transform (see e.g. [10] and references therein). Moreover, it is not yet even fully understood whether widely observed high SNR (more precisely, high signal power) limits is a fundamental feature of the nonlinear channels or it is rather a consequence of using linear approaches in such channels. Overall, it is important to develop better understanding of the basic properties of nonlinear communication channels.

We will use here a particular model of the nonlinear communication channel that allows us to study in more detail impact of nonlinearity on transmission of conventional signals. Namely, we use as master model the nonlinear Schrödinger equation (NLSE) that under certain conditions and within some limits describes the propagation of a signal down an optical fiber (see e.g. [10]-[15] and discussions therein).

$$
i \frac{\partial Q}{\partial Z}-\frac{\beta_{2}}{2} \frac{\partial^{2} Q}{\partial T^{2}}+\gamma|Q|^{2} Q=0
$$

Here $Q$ is a complex envelope field that describes the optical signal, $Z$ is a distance (e.g. in $k m$ ), $T$ is time (e.g. in $p s$ ), $\beta_{2}$ (in $p s^{2} / \mathrm{km}$ ) is the group velocity dispersion parameter and $\gamma$ (in $W^{-1} \mathrm{~km}^{-1}$ ) is the nonlinear Kerr coefficient. Again, we would like to stress that we consider just a model and realistic fiber channels have more complex basic equations. Applicability of the NLSE in the fiber-optic communications has been discussed in a number of previous works (see e.g. [10], [12], [13], [15]. We use here the NLSE-channel to illustrate a particular new feature introduced by nonlinearity in signal analysis. It is convenient to introduce the characteristic time scale, e.g. carrier pulse width or symbol interval $T_{0}$, dispersion length $L_{D}=T_{0}^{2} /\left|\beta_{2}\right|$ and characteristic power $P_{0}=1 /\left(\gamma L_{D}\right)$ to define corresponding non-dimensional variables $t=T / T_{0}, z=Z / L_{D}$ and $q=Q / \sqrt{P_{0}}$ in which the dimensionless nonlinear Schrödinger equation reads:

$$
i \frac{\partial q}{\partial z}+\frac{1}{2} \frac{\partial^{2} q}{\partial t^{2}}+|q|^{2} q=0
$$

In the context of fiber-optic, we consider here the case of the so-called anomalous dispersion $\beta_{2}<0$, when general solutions of this equation can include both the dispersive (linear-like) waves and the coherent localised (in time) structures - solitons. Any initial signal evolving according to this master model can be presented as a nonlinear superposition of dispersive waves and soliton(s).

In general, nonlinear partial differential equations are difficult to solve, not many analytical solutions are possible, and 
numerical modelling is the most typical way to analyse them. However, the nonlinear Schrödinger equation (2) belongs to the class of the so-called integrable nonlinear partial differential equations. In 1972, Zakharov and Shabat demonstrated that the NLSE can be integrated by the inverse scattering transform (IST) method [1], also known nowadays as the nonlinear Fourier transform (NFT). Direct NFT corresponds to solving the Zakharov-Shabat spectral problem (ZSSP). We consider here ZSSP problem for the initial field $q(t, z=0)$ :

$$
\left\{\begin{aligned}
-\partial_{t} \psi_{1}+q(t, 0) \psi_{2} & =i \xi \psi_{1} \\
\partial_{t} \psi_{2}+q^{*}(t, 0) \psi_{1} & =i \xi \psi_{2}
\end{aligned}\right.
$$

where $q(t, 0)=q_{0}(t)$ is the "potential" — initial distribution of the signal to be transmitted, $\psi_{1,2}$ is a vector eigenfunction and $\xi=\lambda+i \eta$ - spectral parameter defined on a complex plane.

In the case of anomalous dispersion $\left(\beta_{2}<0\right)$ the initial localized in time signal distribution $q(t, z=0)$ might have both continuous and discrete spectrum of the system (3). The continuous spectrum $r(\lambda)$ fills the real axis of the $\xi$-plane and corresponds to the dispersive wave component, being the direct analogue to Fourier transform and converging into it at low signal powers. The discrete spectrum eigenvalues $\xi_{n}$, located in the upper complex half-plane $(\eta>0)$, correspond to soliton solutions of the NLSE. The discrete spectrum consists of the set of complex-valued eigenvalues $\xi_{n}$ having a positive imaginary part together with complex-valued norming constants $r_{n}$. Solution of the ZSSP gives full description of the nonlinear spectrum. Parameters of the nonlinear spectrum are changed in a trivial way during evolution along the NLSE channel, allowing one to determine signal $q(t, z)$ at an arbitrary distance from initial distribution at $z=0$. In this work we calculate nonlinear spectrum for a range of conventional initial signals to determine probability of having discrete (soliton) components in such signals. In case soliton component is present in the initial signal it will stay during propagation in the NLSE channel.

\section{Methodology OF COMPUTATION OF NONLINEAR SPECTRA}

In this section we describe methodology used to numerically calculate nonlinear spectra of standard telecom signals. To test the accuracy of the numerical codes one can use a number of exact analytical results with known nonlinear spectrum, see for detail e.g. [16]-[18]. It is useful to recall an important result for the number of discrete eigenvalues (solitons) containing in a rectangular pulse without phase modulation:

$$
N=\operatorname{int}\left[1 / 2+L_{1}(q) / \pi\right]
$$

where int $[\ldots]$ means integer part of the expression and $L_{1}$ norm is defined as $L_{1}(q)=\int_{-\infty}^{+\infty}|q(t)| d t$. However, for more complex signals, there is no similar exact analytical results and numerical modelling has to be used to solve Zakharov-Shabat spectral problem (3) to determine the nonlinear spectrum.

In this paper we focus on the total number of the discrete eigenvalues in a given signal rather than on specific features of the eigenvalue parameters. Therefore, we apply robust methods that determine number of poles in the complex plane basing on the Cauchy theorem (see, e.g. [19]), which associates the number of zeros with a complete phase shift of one of the scattering coefficient in the Zakharov-Shabat spectral problem (3) (for details see [19]-[21]):

$$
N=\left.\frac{1}{2 \pi} \operatorname{Arg}(a(\xi))\right|_{-\infty} ^{+\infty},
$$

where the spectral parameter $\xi$ takes values from $-\infty$ to $+\infty$ on the real axe. A similar approach also can be used to determine the exact location of the discrete eigenvalues, see for detail [21].

Here we study the existence of a soliton component in two standard return-to-zero optical signals: (i) orthogonal frequency-division multiplexing (OFDM) with multiple carrier frequencies and (ii) wavelength-division multiplexing (WDM) where different optical carriers transfer data in a single optical fiber by using different wavelengths. For both formats, without loss of generality, we focus here on two types of popular modulation formats: quadrature amplitude modulation (QAM) and phase-shift keying (PSK) and consider a single OFDM and WDM symbol (i.e. assuming burst mode transmission with well separated symbols). An information-bearing signal can be treated as a random process in which signal characteristics experience statistical variations that depend on modulation formats and coding [22]. For any such statistical realisation of a return-to-zero signal we can compute nonlinear spectrum using the ZSSP.

As we already mentioned above, the main results of this work do not require the calculation of the signal propagation along the $z$ coordinate. We want to emphasize this nontrivial fact. The existence of solitons depends only on the initial distribution of the field, and the number of solitons does not change in the process of propagation. Moreover, the accompanying characteristics of the discrete and continuous spectrum (so-called scattering data) change in a trivial way [10]. The propagation dynamics of the field is considered in this paper only to assess the system performance using the parameter $\mathrm{Q}^{2}$-factor, which measures the quality of an transmission signal. The $\mathrm{Q}^{2}$-factor value is extrapolated from the conventional error vector magnitude (EVM) function [23] as $Q^{2}=1 / E V M^{2}$ using transmission of $2^{14}$ symbols (OFDM or WDM) per run. At the receiver, the chromatic dispersion was fully compensated and an algorithm based on the 4thpower Viterbi-Viterbi method was used for phase estimation.

\section{SOLITON CONTENT IN THE CONVENTIONAL OPTICAL SIGNALS}

Now we analyse the probability of occurrence of solitons in the OFDM and WDM symbol defined as the ratio of the number of symbols containing discrete eigenvalues (corresponding to solitons) of the Zakharov-Shabat spectral problem to the total number of the examined symbols (statistics based on random input data). In other words, we are not interested in the exact number of solitons in the signal, but rather in their existence in the given symbol. Our goal here is to demonstrate that appearance of solitons in the standard optical signals is not something exotic, but rather is a general situation 
128 subcarriers

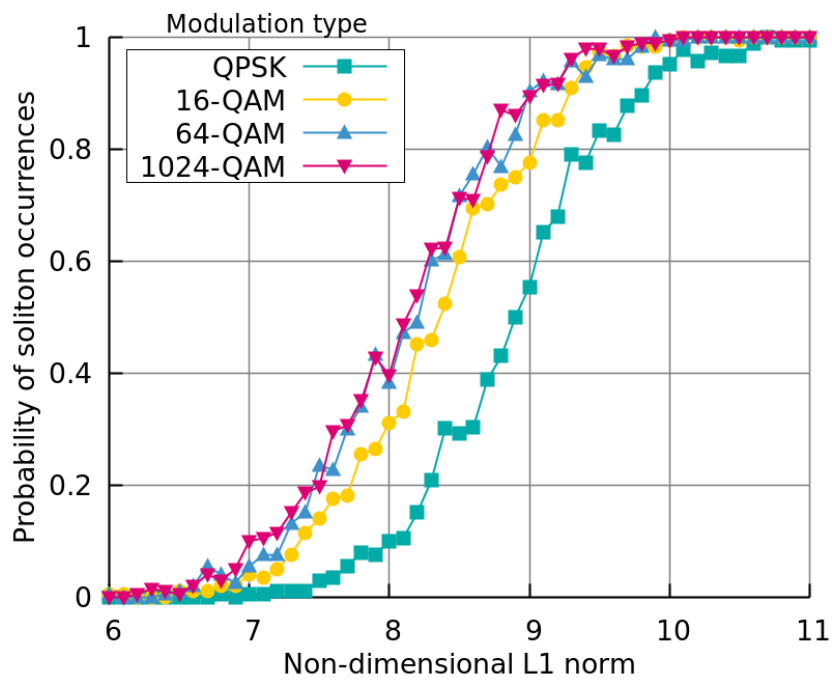

Fig. 1. Average number of occurred discrete values (solitons) versus the $L_{1}$ norm for OFDM signals with 128 subcarriers and QPSK, 16-QAM, 64-QAM and 1024-QAM modulations. The threshold for appearance of the soliton in the non-modulated rectangular pulse, calculated using the formula (4), is $L_{1}=1.57$ and lies on the left well outside the boundaries of the graph

that can occur even at practically used power levels. We did verify that number of solitons does not depend on increase of the computational grid and the FFT size (temporal signal discretization). For numerical simulations we use the following typical fibre parameters: group velocity dispersion parameter $\beta_{2}=-21.5$ (in $p s^{2} / \mathrm{km}$ ) and the nonlinear Kerr coefficient $\gamma=1.27$ (in $W^{-1} \mathrm{~km}^{-1}$ ).

\section{A. OFDM signal}

First, consider the OFDM signal, that presents a sum of independent sub-carriers:

$$
s(t)=\sum_{k=0}^{M-1} X_{k} e^{i 2 \pi k t / T}, 0 \leq t<T,
$$

here $T$ be a symbol interval, $M$ is the number of subcarriers (each corresponds to a frequency in the Fourier transform) and $X_{k}$ corresponds to digital data. In practice, the full number of subcarriers is $2^{p}$ to use FFT algorithm. In this paper the full FFT size is 1024 and number of sub-carriers $M$ is changing from 128 to 1024. In the real world units, we examine OFDM symbol with $10 \mathrm{~ns}$ symbol duration and QPSK, 16-, 64- or 1024-QAM modulation. Average signal power varies (in the dimension units) from -21 to $-7 \mathrm{dBm}$.

In our analysis the varying parameters and characteristics are: modulation type, $L_{1}$ norm or average power $P_{\text {ave }}$ (which corresponds to $L_{2}$ norm) and number of sub-carriers $M$. We examine the probability of solitons occurrence depends on these parameters. For each fixed parameter set we accumulate statistics on the number of realisations by varying input digital data. Each graph point corresponds to 200 statistical measurements.

Figure 1 shows the probability of the occurrence of solitons in OFDM symbol with 128 subcarriers versus the $L_{1}$ norm

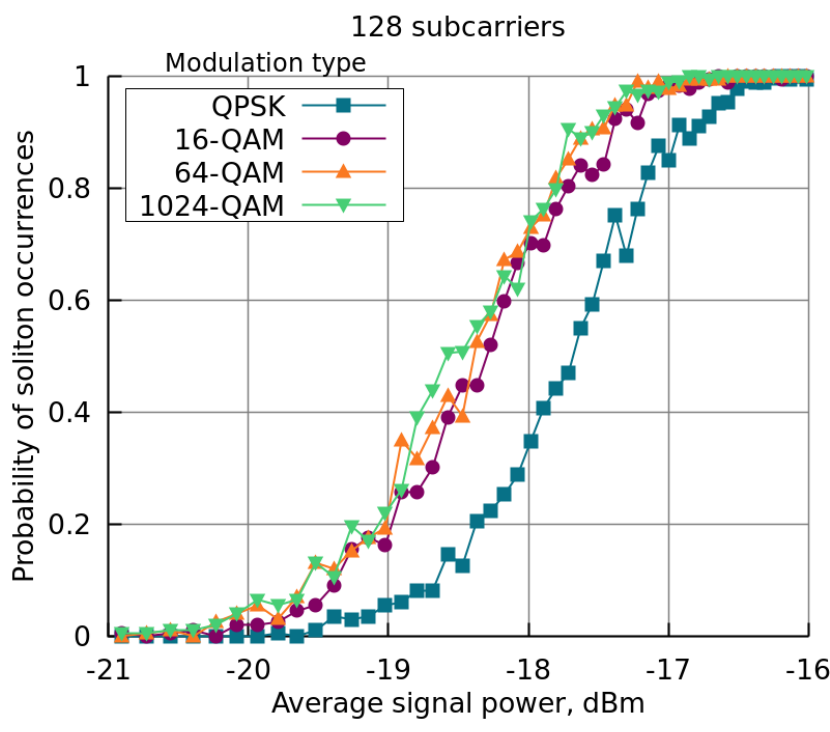

Fig. 2. Average number of solitons embedded into OFDM symbol with 128 sub-carriers and QPSK, 16-, 64- and 1024-QAM modulations versus average signal power.

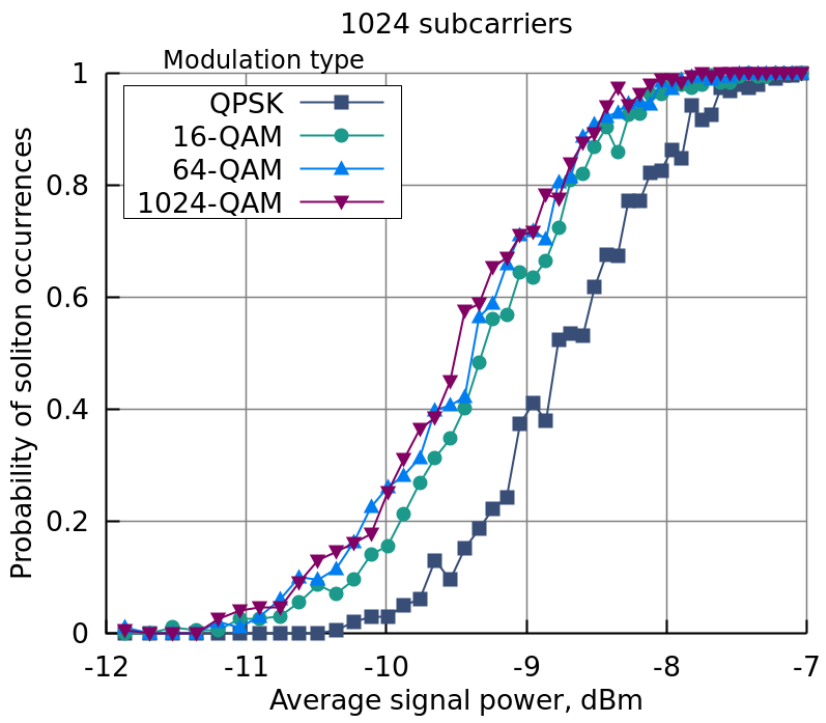

Fig. 3. Average number of solitons embedded into OFDM symbol with 1024 subcarriers and QPSK, 16-, 64- and 1024-QAM modulations versus average signal power.

value for several modulation formats. This and subsequent results show that the probability of the soliton occurrence for signals with the same $L_{1}$ norm or $P_{\text {ave }}$ value slightly increases with the number of constellation points in the corresponding modulation format and saturates at high number of levels.

One can see the same trend in Fig. 2 and 3. These figures demonstrate that with an increase of the order of the constellation diagram (therefore number of bits of information encoded in one symbol) the required average signal power for the existence of solitons decreases. This fact is clearly visible when comparing QPSK and higher-order modulation formats.

Figures 2 and 3 illustrate how the probability of occurrence of the soliton content in the OFDM signal is growing with 


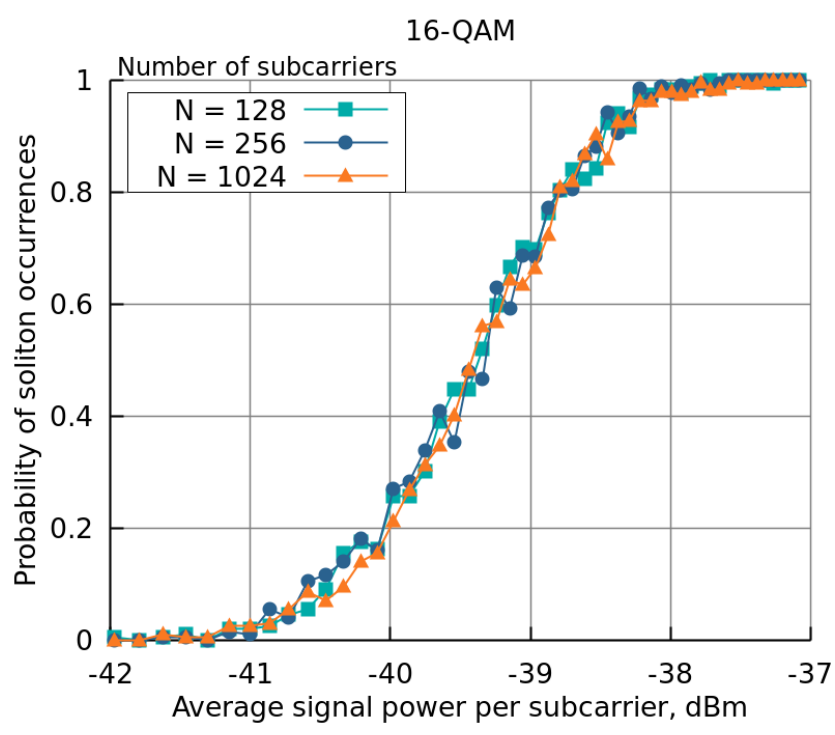

Fig. 4. Average number of solitons embedded into OFDM symbol with 16QAM modulations versus average signal power per one subcarrier.

the increase of the average power $P_{a v e}$. The graphs indicate that the power required for the existence of solitons increases with the number of subcarriers. This effect can be understood from Fig. 4, which shows a similar probability, but versus the average power divided by total number of subcarriers. It is seen that the probability of soliton occurrence is determined by the power density (power per subcarrier). We observe a similar effect for other types of modulation. The specific average power range per subcarrier channel within which soliton occurrence changes from being a rare event to highly probable common situation is approximately from $-42 \mathrm{dBm}$ to $-37 \mathrm{dBm}$. Based on this observation, we can predict the occurrence threshold for other numbers of subcarriers.

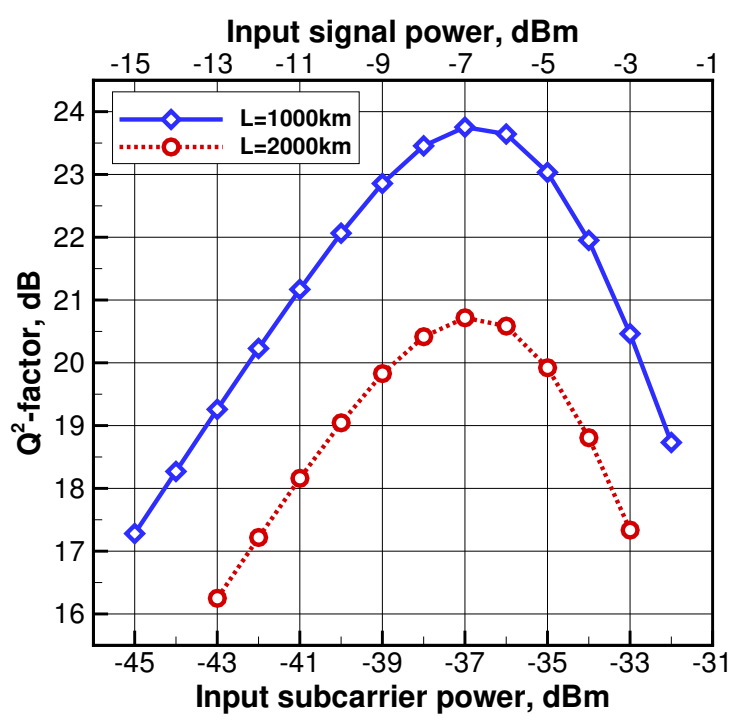

Fig. 5. The dependence of the $\mathrm{Q}^{2}$-factor on the average input power per one subcarrier (below) or total power (above) for the OFDM signal with 16-QAM, 1024 subcarriers and 10 ns symbol duration.
As expected, for a complex signal, the level of $L_{1}$ and $L_{2}$ norms (and hence $P_{a v e}$ ), at which soliton component appears in the signal, is higher than for a simple rectangular signal. This is consistent with the criteria (15) discussed in the Appendix B, which can also be used for a estimate of the threshold.

The interesting observation for OFDM signal is that it does not require too high signal power, for soliton component to become an inherent part of the OFDM symbol. For some parameter sets the soliton component arises at rather practical levels of a signal power conventional for telecom applications. To illustrate this fact, a simulation of a $1000 \mathrm{~km}$ and $2000 \mathrm{~km}$ transmission links for the NLSE channel, with noise estimated from the ideal distributed Raman amplification scheme with continuous amplified spontaneous emission generation (see [10], [14] for detail), was performed. The transmission link characteristics were specified above in Section III. As an input, we used OFDM signal with 16-QAM, 1024 subcarriers and 10 ns symbol duration ( $2^{14}$ OFDM symbols per run). Figure 5 shows the dependence of the $\mathrm{Q}^{2}$-factor on the average input power per one subcarrier (below) and on total average power of the symbol (above) for such system. The results demonstrate that at the power levels optimal for transmission (average power around $-37 \mathrm{dBm}$ ) there is a probability close to one to have a soliton component in signals of this type, according to Fig. 4.

\section{B. WDM signal with return-to-zero carrier pulse}

In this section we examine occurrence of solitons in conventional WDM signals with return-to-zero carrier pulse. A single WDM symbol has a form similar to OFDM signal considered in previous section:

$$
s(t)=\sum_{k=1}^{M} C_{k} e^{i \omega_{k} t} f(t), 0 \leq t<T,
$$

where $M$ is a number of WDM channels, $\omega_{k}$ is a carrier frequency of the $k$-th channel, $C_{k}$ corresponds to the digital data in $k$-th channel and $T$ is a symbol interval. $f(t)$ is a waveform of a return-to-zero carrier pulse, which in this work, without loss of generality, is defined (in the normalized form) as

$$
f(t)=\left\{\begin{array}{c}
\frac{1}{2}\left[1-\cos \left(\frac{4 \pi t}{T}\right)\right], \\
0 \leq t \leq \frac{T}{4} \text { or } \frac{3 T}{4} \leq t \leq T \\
1, \frac{T}{4}<t<\frac{3 T}{4}
\end{array}\right.
$$

Similar to the OFDM signal, we consider QPSK, 16-QAM, 64-QAM and 1024-QAM modulation formats. In the analysis, we use the following standard WDM parameters (in dimensional variables): $100 \mathrm{ps}$ symbol duration and $25 \mathrm{GHz}$ channel spacing. For QPSK, 16-QAM, 64-QAM and 1024QAM modulations these parameters give a channel bit rate of 20,40, 60 and $100 \mathrm{Gbit} / \mathrm{s}$ per polarization and spectral efficiency of $1.6,3.2,4.8,8 \mathrm{bits} / \mathrm{s} / \mathrm{Hz}$, respectively.

As in previous section we examine single isolated return-tozero WDM symbol and analyse the probability of the soliton occurrence in the signal depending on power, modulation type 


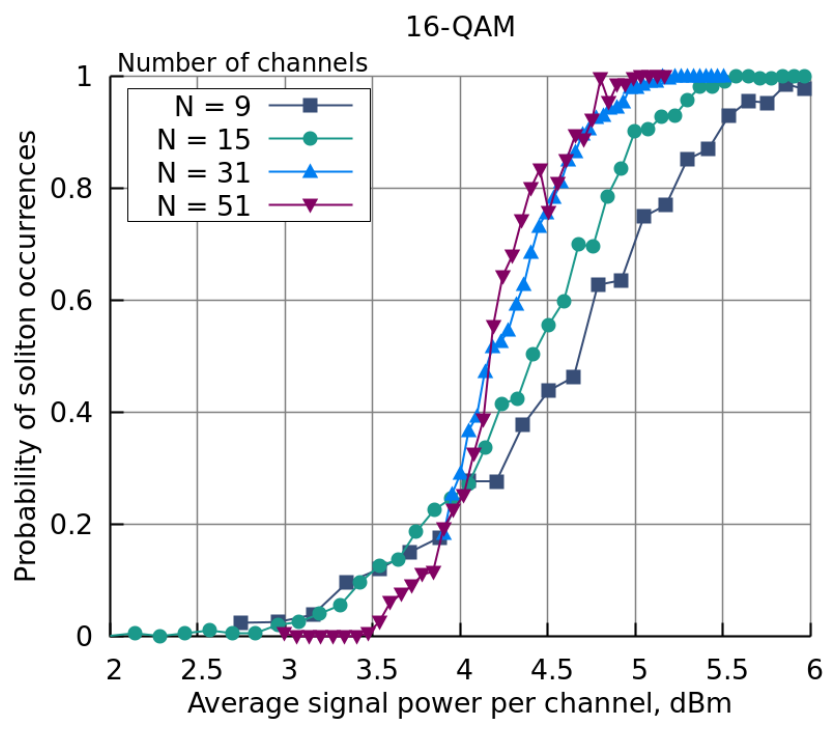

Fig. 6. Probability of occurrence of discrete eigenvalues (solitons) versus average power per channel for WDM signals with 16-QAM modulation and $9,15,31$ and 51 channels.

and number of WDM channels. We accumulated statistics for different sets of WDM symbol parameters by varying $C_{k}$ and each point on the graphs below corresponds to 200 statistical realisations. We would like to note that in the following figures we use the average power per channel. An increase in the number of channels, the interval in which transition from lower probability of having solitons to almost certain presence of soliton content in the symbol slightly decreases with increasing number of channels. Note that corresponding intervals for the total power obviously increases with growing number of channels. A similar tendency is observed for other modulation formats (QPSK, 64-QAM, 1024-QAM).

Figure 7 shows how the probability of the soliton occurrence in a WDM signal with thirteen optical channels, as a function of the average signal power $P_{\text {ave }}$ per channel. Here, similar to OFDM case the transition from the regime with low probability of soliton content in WDM symbols, to the situation when solitons are highly probable part of the signal, occurs in a narrow interval of $3-4 \mathrm{dBm}$ for 16-QAM, 64-QAM, and 1024-QAM and $1 \mathrm{dBm}$ for QPSK.

There are many similarities between WDM and OFDM cases: Fig. 7 illustrates that for WDM signals with QPSK modulation it requires more average power per channel for the occurrence of soliton component than for other (high-order) modulation formats. For example, with $6 \mathrm{dBm}$ in QPSKmodulated symbols, the probability of soliton existence is $2.5 \%$, while for 16-, 64- and 1024-QAM, the probability is almost $100 \%$. The $50 \%$ probability of the soliton occurrence in symbols is achieved at $6.46 \mathrm{dBm}$ for QPSK, $4.47 \mathrm{dBm}$ for 16QAM, $3.88 \mathrm{dBm}$ for 64-QAM and $3.94 \mathrm{dBm}$ for 1024-QAM. We would like to stress that for smaller powers (down to the power defined in the criteria (15) in the Appendix B), there is also a (rare) probability of occurrence of discrete eigenvalues in nonlinear spectrum.

For WDM signals, optimal transmission powers are lower

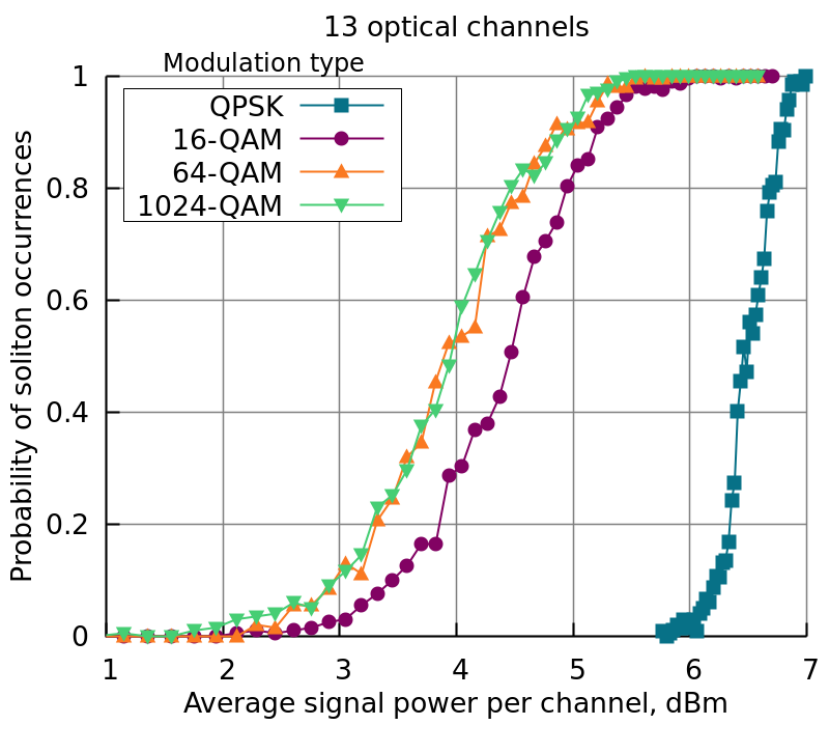

Fig. 7. Probability of occurrence of discrete eigenvalues (solitons) embedded into conventional WDM symbol with 13 channels and QPSK, 16-QAM, 64QAM and 1024-QAM modulations versus average signal power per channel.

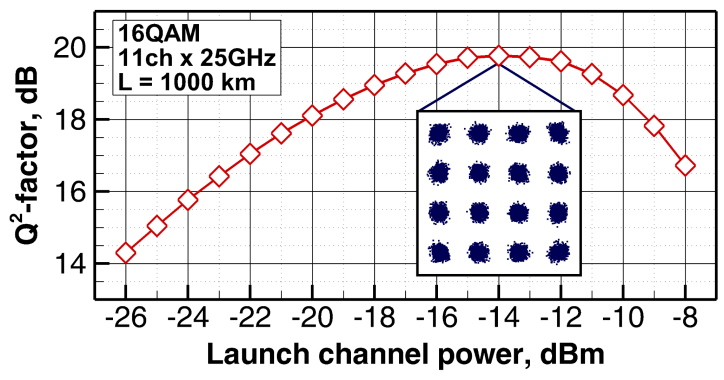

Fig. 8. The dependence of the $\mathrm{Q}^{2}$-factor on the average input power per channel for the WDM signal with 16-QAM modulation, 11 WDM channels and 100 ps symbol duration.

than level of powers where all symbols have solitons. We performed a similar simulation of the a $1000 \mathrm{~km}$ transmission links. The transmission link characteristics (including noise) were specified above in Section III. As an input, we consider WDM signal with 16-QAM, 11 optical channels with 25 GHz channel spacing and 100 ps symbol duration $\left(2^{14} \mathrm{WDM}\right.$ symbols per run). Fig. 8 depicts the dependence of the $\mathrm{Q}^{2}$ factor on the average input power per spectral channel. It is seen that at optimal power levels for transmission, either there are no solitons or solitons with very low probability in the considered WDM signals. However, we did not consider low probability events in this work. Evidently, results for soliton content occurrence for WDM depend on the carrier pulse shape and other specific signal features.

\section{CONCLUSIONS}

Nonlinear channels have properties rather different from linear communication ones. Here we use a particular, albeit important example of the NLSE-channel to illustrate that soliton components might be an inherent part of the conventional signals transmitted in such communication channel. The 
presence of soliton component embedded into the conventional OFDM symbol potentially can impact the transmission of the combined signal. Focus of this paper is on attracting attention to this fact rather then a comprehensive analysis of its consequences for signal coding, modulation and processing and its potential to improve the performance of optical communication systems. We anticipate that similar effects will feature in the integrable nonlinear channel model taking into account polarisations [24], [25]. Additional analysis is required to understand in what way the presence of a soliton component in conventional signals affects transmission and how it can be exploited in practice. Note that even for quasilinear signal transmission statistical fluctuations in the plane $(z, t)$ that occur due to information content of a signal might affect quality of transmission, see e.g. [26].

Conventional signal processing and modulation methods have been designed and developed for linear communication channels. Considering the NLSE-channel we have demonstrated here that a traditional OFDM signal statistically contains soliton components at powers corresponding to lowest BER. This observation indicates that transmission in a nonlinear channel substantially changes the whole paradigm of signal modulation and processing. Our results show that NFT analysis might be useful not only to special inherently soliton-based systems and signals [13], but also for conventional communication formats that traditionally are not linked to the soliton concept and techniques. We believe that our results indicate that application of the detection and processing methods developed for linear channels might be not optimal for nonlinear communication channels.

The results thus obtained are compatible with criteria (15) and show that both WDM and OFDM signals contain solitons upon reaching a certain power levels. Despite the fact that at optimal powers in conventional WDM signals solitons do not occur or appear rarely, we can use NFT for such systems to account for nonlinear distortions.

Considered NFT processing of signals require efficient numerical algorithms such as, e.g. [27]-[29]. These methods will be useful for application of NFT in other areas beyond optical communications.

\section{ACKNOWLEDGMENT}

This work was supported by the Russian Science Foundation (Grant No. 17-72-30006, all authors) and, partially, by the EPSRC grant TRANSNET (SKT).

\section{APPENDIX A}

EXAMPLES OF DISCRETE SPECTRA OF PARTICULAR SIGNAL

\section{A. Hyperbolic secant pulse}

One of the known exact solutions of ZSSP was found by Satsuma and Yajima in 1974 [30]: $q(t, 0)=A \operatorname{sech}(t)$. Here amplitude $A$ is a real positive number. The discrete spectrum of the problem (3) is determined by the following expression:

$$
\xi_{n}=i\left(A+\frac{1}{2}-n\right),
$$

where $\mathrm{n}$ is a positive integer and always $n<A+\frac{1}{2}$ to satisfy the condition that all eigenvalues are in the upper complex half-plane.

\section{B. Rectangular pulse}

Other well-known analytical solution is a rectangular pulse

$$
q(t, 0)=\left\{\begin{array}{cl}
A, & 0 \leq t \leq 1 \\
0, & \text { otherwise }
\end{array}\right.
$$

New eigenvalue appears whenever $A=\pi\left(n-\frac{1}{2}\right)$, where $n$ is a positive integer. The total number of discrete eigenvalues can be calculated by the formula

$$
N=\operatorname{int}\left[1 / 2+L_{1}(q) / \pi\right]
$$

int $[. .$.$] means integer part of the expression. To characterize$ the signals, we use the (non-dimensional) signal $L_{1}$ and $L_{2}$ norms defined by the following expressions:

$$
\begin{aligned}
L_{1}(q) & =\int_{-\infty}^{+\infty}|q(t)| d t, \\
L_{2}(q) & =\int_{-\infty}^{+\infty}|q(t)|^{2} d t .
\end{aligned}
$$

Evidently, the $L_{2}$ norm corresponds to the signal energy. Average signal power is linked to the $L_{2}$ norm (in dimension units) as following: $P_{\text {ave }}=L_{2} / T$.

\section{General criteria of the existence of a discrete spectrum}

The exact condition for the existence of discrete eigenvalues was found in 2003 by Klaus and Shaw [31]. They showed that for initial complex distribution $q(t, 0)$ the Zakharov-Shabat system (3) has no discrete eigenvalues when:

$$
L_{1}(q(t, 0)) \leq \frac{\pi}{2}
$$

This criterion is strictly related to the $L_{1}$ norm, however, it can be extended to the $L_{2}$ norm. Using the definition of the $L_{1}$ and $L_{2}$ norms, as well as a standard inequality for the arithmetic and geometric means, we can derive for the signals considered at the finite intervals $T$ :

$$
L_{2}>\frac{\pi^{2}}{4 T}
$$

which we can rewrite in real world units as

$$
P_{\text {ave }}>\frac{\left|\beta_{2}\right| \pi^{2}}{4 \gamma T^{2}},
$$

where $P_{\text {ave }}$ is a average signal power and $T$ - symbol interval. Figure 9 shows how criteria (15) depends on the symbol interval. One can see that with a decrease of the symbol interval, the average power at which discrete eigenvalues can appear is dramatically increasing. The graph also shows two levels: $100 \mathrm{ps}$ and $10 \mathrm{~ns}$, which we examined in this work. For these two cases, the average power levels, defined by the formula (15), are at $6 \mathrm{dBm}$ and $-30 \mathrm{dBm}$, respectively.

Finally, Fig. 10 depicts a typical example of the discrete nonlinear spectrum of particular OFDM signal with 128 subcarriers, QPSK modulation and an average signal power of $-16 \mathrm{dBm}$ and a WDM signal with 13 channels, QPSK modulation and average power per channel of $6.35 \mathrm{dBm}$. 


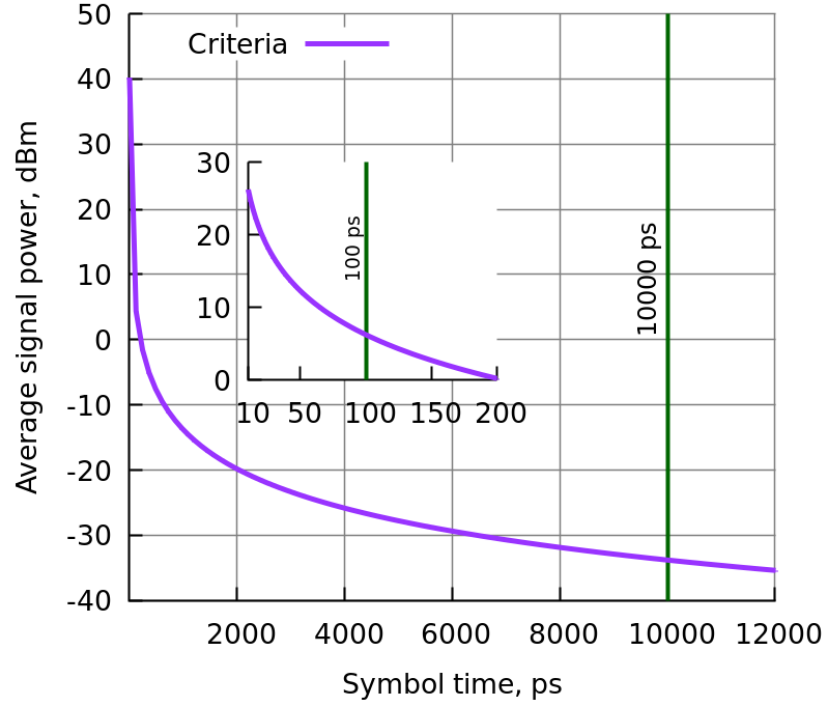

Fig. 9. General criteria of the existence of a discrete spectrum defined by the formula (15).

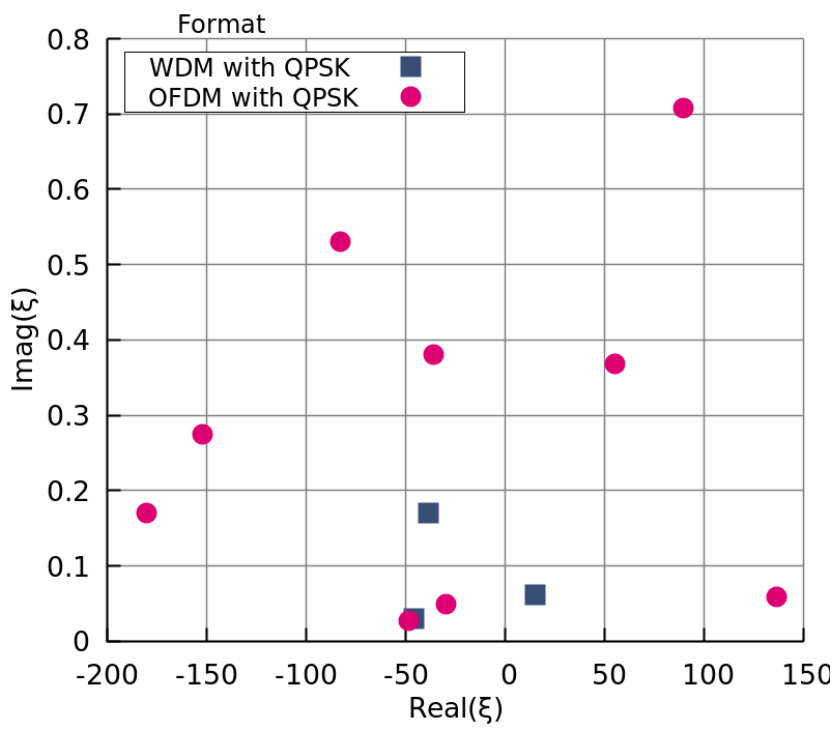

Fig. 10. Example of the nonlinear discrete spectrum for OFDM signal with QPSK modulation, 128 subcarriers and an average signal power of $-16 \mathrm{dBm}$ and WDM signal with QPSK modulation, 13 channels and average power per channel of $6.35 \mathrm{dBm}$.

\section{REFERENCES}

[1] V. E. Zakharov and A. B. Shabat, "Exact theory of two-dimensional self-focusing and one-dimensional self-modulation of waves in nonlinear media," Soviet Physics JETP, vol. 34, no. 1, p. 62, 1972.

[2] E. Ip and J. M. Kahn, "Compensation of dispersion and nonlinear impairments using digital backpropagation," Journal of Lightwave Technology, vol. 26, no. 20, pp. 3416-3425, 2008.

[3] D. Marsella, M. Secondini, and E. Forestieri, "Maximum likelihood sequence detection for mitigating nonlinear effects," Journal of Lightwave Technology, vol. 32, no. 5, pp. 908-916, 2014.

[4] L. B. Du, D. Rafique, A. Napoli, B. Spinnler, A. D. Ellis, M. Kuschnerov, and A. J. Lowery, "Digital fiber nonlinearity compensation: Toward 1-tb/s transport," IEEE Signal Processing Magazine, vol. 31, no. 2, pp. 46-56, March 2014

[5] F. P. Guiomar, J. D. Reis, A. L. Teixeira, and A. N. Pinto, "Mitigation of intra-channel nonlinearities using a frequency-domain volterra series equalizer," Opt. Express, vol. 20, no. 2, pp. 1360-1369, Jan 2012.
[6] R. Dar, M. Feder, A. Mecozzi, and M. Shtaif, "Inter-channel nonlinear interference noise in wdm systems: Modeling and mitigation," Journal of Lightwave Technology, vol. 33, no. 5, pp. 1044-1053, March 2015.

[7] A. Ellis and M. Sorokina, Optical Communication Systems: Limits and Possibilities. Jenny Stanford Publishing, 2019.

[8] O. Vassilieva, I. Kim, and T. Ikeuchi, "Enabling technologies for fiber nonlinearity mitigation in high capacity transmission systems," $J$. Lightwave Technol., vol. 37, no. 1, pp. 50-60, Jan 2019.

[9] M. Cvijetic and I. Djordjevic, Advanced Optical Communication Systems and Networks, ser. Artech House applied photonics series. Artech House, 2013.

[10] S. K. Turitsyn, J. E. Prilepsky, S. T. Le, S. Wahls, L. L. Frumin, M. Kamalian, and S. A. Derevyanko, "Nonlinear fourier transform for optical data processing and transmission: advances and perspectives," Optica, vol. 4, no. 3, pp. 307-322, 2017.

[11] G. P. Agrawal, The Nonlinear Fiber Optics, 4th ed. Academic press, 2007.

[12] A. Hasegawa and Y. Kodama, "Guiding-center soliton in optical fibers," Optics Lett., vol. 15, pp. 1443-1445, 1990.

[13] L. F. Mollenauer and J. Gordon, Solitons in optical fiber. Academic Press, 2006

[14] J. D. Ania-Castañón, T. J. Ellingham, R. Ibbotson, X. Chen, L. Zhang, and S. K. Turitsyn, "Ultralong raman fibre lasers as virtually lossless optical media," Phys. Rev. Lett., vol. 96, p. 023902, 2006.

[15] R.-J. Essiambre, G. Kramer, P. J. Winzer, G. J. Foschini, and B. Goebel, "Capacity limits of optical fiber networks," Journal of Lightwave Technology, vol. 28, no. 4, pp. 662-701, 2010.

[16] S. Manakov, "Nonlinear fraunhofer diffraction," Soviet Physics JETP, vol. 38, no. 4, p. 693, 1974.

[17] Y. S. Kivshar, "On the soliton generation in optical fibres," Journal of Physics A: Mathematical and General, vol. 22, no. 3, p. 337, 1989.

[18] J. Burzlaff, "The soliton number of optical soliton bound states for two special families of input pulses," Journal of Physics A: Mathematical and General, vol. 21, no. 2, p. 561, 1988.

[19] S. Burtsev, R. Camassa, and I. Timofeyev, "Numerical algorithms for the direct spectral transform with applications to nonlinear schrdinger type systems," Journal of computational physics, vol. 147, no. 1, pp. 166-186, 1998.

[20] G. Boffetta and A. R. Osborne, "Computation of the direct scattering transform for the nonlinear schroedinger equation," Journal of computational physics, vol. 102, no. 2, pp. 252-264, 1992.

[21] A. Vasylchenkova, J. E. Prilepsky, and S. K. Turitsyn, "Contour integrals for numerical computation of discrete eigenvalues in the zakharovshabat problem," Optics Lett., vol. 43, no. 15, pp. 3690-3693, 2018.

[22] J. G. Proakis, Digital Communications, 4th ed. McGraw-Hill, New York, 2000.

[23] R. Schmogrow, B. Nebendahl, M. Winter, A. Josten, D. Hillerkuss, S. Koenig, J. Meyer, M. Dreschmann, M. Huebner, C. Koos, J. Becker, W. Freude, and J. Leuthold, "Error vector magnitude as a performance measure for advanced modulation formats," IEEE Photonics Technology Letters, vol. 24, no. 1, pp. 61-63, 2012.

[24] S. V. Manakov, "On the theory of two-dimensional stationary selffocusing of electromagnetic waves," Soviet Physics-JETP, vol. 38, no. 2, pp. 248-253, 1974.

[25] S. Gaiarin, A. M. Perego, E. Porto da Silva, F. Da Ros, and D. Zibar, "Dual polarization nonlinear fourier transform-based optical communication system: supplementary material," Optica, 2019.

[26] S. Derevyanko, A. Redyuk, S. Vergeles, and S. K. Turitsyn, "Visualisation of extreme value events in optical communications," APL Photonics, vol. 3, p. 060801, 2018.

[27] S. Wahls and H. V. Poor, "Fast numerical nonlinear fourier transforms," IEEE Transactions on Information Theory, vol. 61, no. 12, pp. 69576974, 2015.

[28] S. Wahls and H. V. Poor, "Fast inverse nonlinear fourier transform for generating multi-solitons in optical fiber," in 2015 IEEE International Symposium on Information Theory (ISIT), June 2015, pp. 1676-1680.

[29] S. Medvedev, I. Vaseva, I. Chekhovskoy, and M. Fedoruk, "Numerical algorithm with fourth-order accuracy for the direct zakharov-shabat problem," Opt. Lett., vol. 44, no. 9, pp. 2264-2267, 2019.

[30] J. Satsuma and N. Yajima, "B. Initial Value Problems of OneDimensional Self-Modulation of Nonlinear Waves in Dispersive Media," Progress of Theoretical Physics Supplement, vol. 55, pp. 284-306, 1974.

[31] M. Klaus and J. K. Shaw, "On the eigenvalues of zakharov-shabat system," J. Math. Anal., vol. 34, no. 4, pp. 759-773, 2003. 